PROCEEDINGS OF THE

AMERICAN MATHEMATICAL SOCIETY

Volume 140, Number 3, March 2012, Pages 1011-102

S 0002-9939(2011)10952-3

Article electronically published on July 21, 2011

\title{
THE DIFFERENTIABLE SPHERE THEOREM FOR MANIFOLDS WITH POSITIVE RICCI CURVATURE
}

\author{
HONG-WEI XU AND JUAN-RU GU
}

(Communicated by Jianguo Cao)

\begin{abstract}
We prove that if $M^{n}$ is a compact Riemannian $n$-manifold and if $R i c_{\min }>(n-1) \tau_{n} K_{\max }$, where $K_{\max }(x):=\max _{\pi \subset T_{x} M} K(\pi), \operatorname{Ric}_{\min }(x):=$ $\min _{u \in U_{x} M} \operatorname{Ric}(u), K(\cdot)$ and $\operatorname{Ric}(\cdot)$ are the sectional curvature and Ricci curvature of $M$ respectively, and $\tau_{n}=1-\frac{6}{5(n-1)}$, then $M$ is diffeomorphic to a spherical space form. In particular, if $M$ is a compact simply connected manifold with $K \leq 1$ and $\operatorname{Ric}_{M}>(n-1) \tau_{n}$, then $M$ is diffeomorphic to the standard $n$-sphere $S^{n}$. We also extend the differentiable sphere theorem above to submanifolds in Riemannian manifolds with codimension $p$.
\end{abstract}

\section{Introduction AND MAIN RESUlts}

In 1989, Shen [14] obtained the following important topological sphere theorem for manifolds of positive Ricci curvature (see also [16]).

Theorem A. Let $M^{n}$ be an $n$-dimensional complete and simply connected manifold. If the sectional curvature satisfies $K_{M} \leq 1$, and the Ricci curvature satisfies $\operatorname{Ric}_{M} \geq(n-1) \delta_{n}$, where

$$
\delta_{n}= \begin{cases}\frac{5}{8}-\frac{3}{8(n-1)} & \text { for even } n, \\ \frac{5}{8}-\frac{3}{4(n-1)} & \text { for odd } n,\end{cases}
$$

then $M$ is homeomorphic to the $n$-sphere $S^{n}$.

A natural question related to Shen's sphere theorems is as follows.

Question. Can one prove a differentiable sphere theorem for manifolds satisfying similar pinching condition?

In 1966, Calabi (unpublished) and Gromoll 8 first investigated the differentiable pinching problem for positive pinched compact manifolds. During the past four decades, there has been much progress on differentiable pinching problems for Riemannian manifolds and submanifolds 1, 2, 5, 16. In 2007, Brendle and Schoen

Received by the editors November 6, 2010 and, in revised form, December 11, 2010.

2010 Mathematics Subject Classification. Primary 53C20; Secondary 53C40.

Key words and phrases. Compact manifolds, differentiable sphere theorem, Ricci curvature, Ricci flow, second fundamental form.

Research supported by the NSFC, grant No. 10771187, 11071211, and the Trans-Century Training Programme Foundation for Talents by the Ministry of Education of China.

(C)2011 American Mathematical Society Reverts to public domain 28 years from publication 
proved a remarkable differentiable sphere theorem [6, 7] for manifolds with quarter pinched curvature in the pointwise sense. Recently Brendle [3] obtained the following striking convergence theorem for the Ricci flow.

Theorem B. Let $\left(M, g_{0}\right)$ be a compact Riemannian manifold of dimension $n(\geq 4)$. Assume that

$$
R_{1313}+\lambda^{2} R_{1414}+R_{2323}+\lambda^{2} R_{2424}-2 \lambda R_{1234}>0
$$

for all orthonormal four-frames $\left\{e_{1}, e_{2}, e_{3}, e_{4}\right\}$ and all $\lambda \in[0,1]$. Then the normalized Ricci flow with initial metric $g_{0}$

$$
\frac{\partial}{\partial t} g(t)=-2 R i c_{g(t)}+\frac{2}{n} r_{g(t)} g(t)
$$

exists for all time and converges to a constant curvature metric as $t \rightarrow \infty$. Here $r_{g(t)}$ denotes the mean value of the scalar curvature of $g(t)$.

Let $M^{n}$ be an $n$-dimensional submanifold in an $(n+p)$-dimensional Riemannian manifolds $N^{n+p}$. Denote by $H$ and $S$ the mean curvature and the squared length of the second fundamental form of $M$, respectively. Let $\bar{K}(\pi)$ be the sectional curvature of $N$ for the tangent 2-plane $\pi\left(\subset T_{x} N\right)$ at the point $x \in N$. Set $\bar{K}_{\max }(x):=\max _{\pi \subset T_{x} N} \bar{K}(\pi)$. Denote by $\overline{\operatorname{Ric}}_{\min }^{(\mathbf{k})}(x)$ the minimum of the $\mathbf{k}$-th Ricci curvature of $N$ at the point $x \in N$. The geometry and topology of the $\mathbf{k}$-th Ricci curvature were initiated by Hartman [10] in 1979 and developed by Wu [18, Shen [14, 15] and others.

In this paper, we investigate the differentiable pinching problem for compact submanifolds in Riemannian manifolds with codimension $p(\geq 0)$. Using Brendle's convergence theorem for the Ricci flow, we prove a differentiable sphere theorem for manifolds with lower bound for Ricci curvature and upper bound for sectional curvature, which is an answer to our question. We first prove the following differentiable sphere theorem for general submanifolds.

Theorem 1.1. Let $M^{n}$ be an $n$-dimensional, $n \geq 3$, complete submanifold in an $(n+p)$-dimensional Riemannian manifold $N^{n+p}$ with codimension $p(\geq 0)$. If

$$
\sup _{M}\left[S-\frac{n^{2} H^{2}}{n-1}-\frac{5}{3}\left(\overline{R i c}_{\min }^{(\mathbf{k})}-\left(\mathbf{k}-\frac{6}{5}\right) \bar{K}_{\max }\right)\right]<0,
$$

for some integer $\mathbf{k} \in[2, n+p-1]$, then $M$ is diffeomorphic to a spherical space form. In particular, if $M$ is simply connected, then $M$ is diffeomorphic to $S^{n}$.

Moreover, we get the following differentiable sphere theorem.

Theorem 1.2. Let $M^{n}$ be an $n$-dimensional, $n \geq 3$, complete submanifold in an $(n+p)$-dimensional Riemannian manifold $N^{n+p}$ with codimension $p(\geq 0)$. If

$$
\sup _{M}\left[S-\frac{5 \sqrt{2}}{3}\left(\overline{\operatorname{Ric}}_{\min }^{(\mathbf{k})}-\left(\mathbf{k}-\frac{6}{5}\right) \bar{K}_{\max }\right)\right]<0,
$$

for some integer $\mathbf{k} \in[2, n+p-1]$, then $M$ is diffeomorphic to a spherical space form. In particular, if $M$ is simply connected, then $M$ is diffeomorphic to $S^{n}$. 
Let $K(\pi)$ be the sectional curvature of $M$ for the tangent 2-plane $\pi \subset T_{x} M$ at the point $x \in M$, and let $\operatorname{Ric}(u)$ be the Ricci curvature of $M$ for the unit tangent vector $u \in U_{x} M$ at $x \in M$. Set $K_{\max }(x):=\max _{\pi \subset T_{x} M} K(\pi), \operatorname{Ric}_{\min }(x):=$ $\min _{u \in U_{x} M} \operatorname{Ric}(u)$. We obtain the following differentiable sphere theorem for Riemannian manifolds with pinched curvatures in the pointwise sense.

Theorem 1.3. Let $M^{n}$ be an $n$-dimensional, $n \geq 3$, compact Riemannian manifold. If Ric $c_{\min }>(n-1) \tau_{n} K_{\max }$, where $\tau_{n}=1-\frac{6}{5(n-1)}$, then $M$ is diffeomorphic to a spherical space form. In particular, if $M$ is simply connected, then $M$ is diffeomorphic to $S^{n}$.

\section{NOTATION AND FORMULAS}

Let $M^{n}$ be an $n$-dimensional submanifold in an $(n+p)$-dimensional Riemannian manifolds $N^{n+p}$. We shall make use of the following convention on the range of indices:

$$
\begin{gathered}
1 \leq A, B, C, \ldots \leq n+p ; 1 \leq i, j, k, \ldots \leq n \\
\quad \text { if } p \geq 1, n+1 \leq \alpha, \beta, \gamma, \ldots \leq n+p .
\end{gathered}
$$

For an arbitrary fixed point $x \in M \subset N$, we choose an orthonormal local frame field $\left\{e_{A}\right\}$ in $N^{n+p}$ such that the $e_{i}$ 's are tangent to $M$. Denote by $\left\{\omega_{A}\right\}$ the dual frame field of $\left\{e_{A}\right\}$. Let

$$
\begin{gathered}
R m=\sum_{i, j, k, l} R_{i j k l} \omega_{i} \otimes \omega_{j} \otimes \omega_{k} \otimes \omega_{l}, \\
\overline{R m}=\sum_{A, B, C, D} \bar{R}_{A B C D} \omega_{A} \otimes \omega_{B} \otimes \omega_{C} \otimes \omega_{D}
\end{gathered}
$$

be the Riemannian curvature tensors of $M$ and $N$, respectively. Denote by $h$ the second fundamental form of $M$. When $p=0, h$ is identically equal to zero. When $p \geq 1$, we set $h=\sum_{\alpha, i, j} h_{i j}^{\alpha} \omega_{i} \otimes \omega_{j} \otimes e_{\alpha}$. The squared norm $S$ of the second fundamental form and the mean curvature $H$ of $M$ are given by

$$
S=\sum_{\alpha, i, j}\left(h_{i j}^{\alpha}\right)^{2}, H=\left|\frac{1}{n} \sum_{\alpha, i} h_{i i}^{\alpha} e_{\alpha}\right| .
$$

Then we have the Gauss equation

$$
R_{i j k l}=\bar{R}_{i j k l}+\left\langle h\left(e_{i}, e_{k}\right), h\left(e_{j}, e_{l}\right)\right\rangle-\left\langle h\left(e_{i}, e_{l}\right), h\left(e_{j}, e_{k}\right)\right\rangle .
$$



$$
\begin{gathered}
\operatorname{Ric}\left(e_{i}\right)=\sum_{j} R_{i j i j}, \quad \operatorname{Ric}_{\min }(x)=\min _{u \in U_{x} M} \operatorname{Ric}(u) ; \\
\overline{\operatorname{Ric}}\left(e_{A}\right)=\sum_{B} \bar{R}_{A B A B}, \overline{\operatorname{Ric}}_{\min }(x)=\min _{u \in U_{x} N} \overline{\operatorname{Ric}}(u) .
\end{gathered}
$$

For any unit tangent vector $u \in U_{x} M$ at the point $x \in M$, let $V_{x}^{\mathbf{k}}$ be a $\mathbf{k}$-dimensional subspace of $T_{x} M$ satisfying $u \perp V_{x}^{\mathbf{k}}$. Choose an orthonormal basis $\left\{e_{i}\right\}$ in $T_{x} M$ such that $e_{j_{0}}=u, \operatorname{span}\left\{e_{j_{1}}, \ldots, e_{j_{\mathbf{k}}}\right\}=V_{x}^{\mathbf{k}}$, where the indices $1 \leq j_{0}, j_{1}, \ldots, j_{\mathbf{k}} \leq n$ 
are distinct from each other. We set

$$
\begin{gathered}
\operatorname{Ric}^{(\mathbf{k})}\left(u ; V_{x}^{\mathbf{k}}\right)=\operatorname{Ric}^{(\mathbf{k})}\left(e_{j_{0}} ; e_{j_{1}}, \ldots, e_{j_{\mathbf{k}}}\right)=\sum_{\mathbf{s}=1}^{\mathbf{k}} R_{j_{0} j_{\mathbf{s}} j_{0} j_{\mathbf{s}}}, \\
\operatorname{Ric}^{(\mathbf{k})}(u)=\min _{u \perp V_{x}^{\mathbf{k}} \subset T_{x} M} \operatorname{Ric}^{(\mathbf{k})}\left(u ; V_{x}^{\mathbf{k}}\right), \\
\operatorname{Ric}_{\min }^{(\mathbf{k})}(x)=\min _{u \in U_{x} M} \operatorname{Ric}^{(\mathbf{k})}(u)=\min _{u \in U_{x} M} \min _{u \perp V_{x}^{\mathbf{k}} \subset T_{x} M} \operatorname{Ric}^{(\mathbf{k})}\left(u ; V_{x}^{\mathbf{k}}\right) .
\end{gathered}
$$

Definition 2.1. We call $\operatorname{Ric}^{(\mathbf{k})}\left(u ; V_{x}^{\mathbf{k}}\right)$ the $\mathbf{k}$-th Ricci curvature of $M$, and $R i c_{\min }^{(\mathbf{k})}(x)$ is called the minimum of the $\mathbf{k}$-th Ricci curvature of $M$ at the point $x \in M$.

For any unit tangent vector $u \in U_{x} N$ at the point $x \in N$, let $V_{x}^{\mathbf{k}}$ be a kdimensional subspace of $T_{x} N$ satisfying $u \perp V_{x}^{\mathbf{k}}$. Choose an orthonormal basis $\left\{e_{A}\right\}$ in $T_{x} N$ such that $e_{A_{0}}=u, \operatorname{span}\left\{e_{A_{1}}, \ldots, e_{A_{\mathbf{k}}}\right\}=V_{x}^{\mathbf{k}}$, where the indices $1 \leq A_{0}, A_{1}, \ldots, A_{\mathbf{k}} \leq n+p$ are distinct from each other. We define the $\mathbf{k}$-th Ricci curvature and the minimum of the $\mathbf{k}$-th Ricci curvature of $N$ at the point $x \in N$ as follows:

$$
\begin{gathered}
\overline{\operatorname{Ric}}^{(\mathbf{k})}\left(u ; V_{x}^{\mathbf{k}}\right)=\sum_{\mathbf{s}=1}^{\mathbf{k}} \bar{R}_{A_{0} A_{\mathbf{s}} A_{0} A_{\mathbf{s}},} \\
\overline{\operatorname{Ric}}^{(\mathbf{k})}(u)=\min _{u \perp V_{x}^{\mathbf{k}} \subset T_{x} N} \overline{\operatorname{Ric}}^{(\mathbf{k})}\left(u ; V_{x}^{\mathbf{k}}\right), \\
\overline{\operatorname{Ric}}_{\min }^{(\mathbf{k})}(x)=\min _{u \in U_{x} N} \min _{u \perp V_{x}^{\mathbf{k}} \subset T_{x} N} \overline{\operatorname{Ric}}^{(\mathbf{k})}\left(u ; V_{x}^{\mathbf{k}}\right) .
\end{gathered}
$$

Denote by $K(\pi)$ the sectional curvature of $M$ for the tangent 2-plane $\pi\left(\subset T_{x} M\right)$ at the point $x \in M$ and by $\bar{K}(\pi)$ the sectional curvature of $N$ for the tangent 2-plane $\pi\left(\subset T_{x} N\right)$ at the point $x \in N$. Set $K_{\min }(x)=\min _{\pi \subset T_{x} M} K(\pi), K_{\max }(x)=$ $\max _{\pi \subset T_{x} M} K(\pi), \bar{K}_{\min }(x)=\min _{\pi \subset T_{x} N} \bar{K}(\pi), \bar{K}_{\max }(x)=\max _{\pi \subset T_{x} N} \bar{K}(\pi)$. Then by Berger's inequality, we have

$$
\left|R_{i j k l}\right| \leq \frac{2}{3}\left(K_{\max }-K_{\min }\right)
$$

for all distinct indices $i, j, k, l$, and

$$
\left|\bar{R}_{A B C D}\right| \leq \frac{2}{3}\left(\bar{K}_{\max }-\bar{K}_{\min }\right)
$$

for all distinct indices $A, B, C, D$.

\section{Proof OF THE THEOREMS}

Theorem 3.1. Let $M^{n}$ be an $n$-dimensional, $n \geq 4$, compact submanifold in an $(n+p)$-dimensional Riemannian manifold $N^{n+p}$ with codimension $p(\geq 0)$. If

$$
S<\frac{5}{3}\left[\overline{\operatorname{Ric}}_{\min }^{(\mathbf{k})}-\left(\mathbf{k}-\frac{6}{5}\right) \bar{K}_{\max }\right]+\frac{n^{2} H^{2}}{n-1},
$$

for some integer $\mathbf{k} \in[2, n+p-1]$, then $M$ is diffeomorphic to a spherical space form. In particular, if $M$ is simply connected, then $M$ is diffeomorphic to $S^{n}$.

Proof. When $p=0$, it is easy to see from (2.2) that

$$
\operatorname{Ric}_{\min }^{(\mathbf{k})} \leq K_{\min }+(\mathbf{k}-1) K_{\max } .
$$


Then we have

$$
K_{\min } \geq R i c_{\min }^{(\mathbf{k})}-(\mathbf{k}-1) K_{\max } .
$$

Suppose $\left\{e_{1}, e_{2}, e_{3}, e_{4}\right\}$ is an orthonormal four-frame and $\lambda \in \mathbb{R}$. From (2.2), (2.8) and (3.1) we obtain

$$
\begin{aligned}
& R_{1313}+R_{2323}-\left|R_{1234}\right| \\
& \geq R i c_{\min }^{(\mathbf{k})}-\sum_{i=3}^{\mathbf{k}+1} R_{i 3 i 3}-\frac{2}{3}\left(K_{\max }-K_{\min }\right) \\
& \geq R i c_{\min }^{(\mathbf{k})}-(\mathbf{k}-2) K_{\max }-\frac{2}{3}\left(\mathbf{k} K_{\max }-R i c_{\min }^{(\mathbf{k})}\right) \\
& \geq \frac{5}{3}\left[R i c_{\min }^{(\mathbf{k})}-\left(\mathbf{k}-\frac{6}{5}\right) K_{\max }\right] .
\end{aligned}
$$

Similarly we get

$$
R_{1414}+R_{2424}-\left|R_{1234}\right| \geq \frac{5}{3}\left[R i c_{\min }^{(\mathbf{k})}-\left(\mathbf{k}-\frac{6}{5}\right) K_{\max }\right] .
$$

From (3.2), (3.3) and the assumption we obtain

$$
\begin{aligned}
& R_{1313}+\lambda^{2} R_{1414}+R_{2323}+\lambda^{2} R_{2424}-2 \lambda R_{1234} \\
& \geq R_{1313}+R_{2323}-\left|R_{1234}\right|+\lambda^{2}\left(R_{1414}+R_{2424}-\left|R_{1234}\right|\right) \\
& \geq \frac{5\left(1+\lambda^{2}\right)}{3}\left[R i c_{\min }^{(\mathbf{k})}-\left(\mathbf{k}-\frac{6}{5}\right) K_{\max }\right] \\
& >0 .
\end{aligned}
$$

Hence the assertion follows from Theorem B.

When $p \geq 1$, it is easy to see from (2.5) that

$$
\overline{R i c}_{\min }^{(\mathbf{k})} \leq \bar{K}_{\min }+(\mathbf{k}-1) \bar{K}_{\max } .
$$

This implies

$$
\bar{K}_{\min } \geq \overline{\operatorname{Ric}}_{\min }^{(\mathbf{k})}-(\mathbf{k}-1) \bar{K}_{\max } .
$$

Setting $S_{\alpha}:=\sum_{i, j=1}^{n}\left(h_{i j}^{\alpha}\right)^{2}$, we have

$$
\left(\sum_{i=1}^{n} h_{i i}^{\alpha}\right)^{2}=(n-1)\left[\sum_{i=1}^{n}\left(h_{i i}^{\alpha}\right)^{2}+\sum_{i \neq j}\left(h_{i j}^{\alpha}\right)^{2}+\frac{\left(\sum_{i=1}^{n} h_{i i}^{\alpha}\right)^{2}}{n-1}-S_{\alpha}\right] .
$$

Note that for $t \neq s$

$$
\begin{aligned}
\left(\sum_{i=1}^{n} h_{i i}^{\alpha}\right)^{2} & \leq(n-1)\left[\left(h_{t t}^{\alpha}+h_{s s}^{\alpha}\right)^{2}+\sum_{i \neq t, s}\left(h_{i i}^{\alpha}\right)^{2}\right] \\
& =(n-1)\left[\sum_{i=1}^{n}\left(h_{i i}^{\alpha}\right)^{2}+2 h_{t t}^{\alpha} h_{s s}^{\alpha}\right] .
\end{aligned}
$$

This together with (3.6) implies

$$
2 h_{t t}^{\alpha} h_{s s}^{\alpha} \geq \sum_{i \neq j}\left(h_{i j}^{\alpha}\right)^{2}+\frac{\left(\sum_{i=1}^{n} h_{i i}^{\alpha}\right)^{2}}{n-1}-S_{\alpha},
$$


where $t \neq s$. Suppose $\left\{e_{1}, e_{2}, e_{3}, e_{4}\right\}$ is an orthonormal four-frame and $\lambda \in \mathbb{R}$. From (2.1), (2.5), (2.9), (3.5) and (3.7) we get

$$
\begin{aligned}
& R_{1313}+R_{2323}-\left|R_{1234}\right| \\
& \geq \overline{R i c}_{\min }^{(\mathbf{k})}-\sum_{A=3}^{\mathbf{k}+1} \bar{R}_{A 3 A 3}-\frac{2}{3}\left(\bar{K}_{\max }-\bar{K}_{\min }\right)+\sum_{\alpha}\left[h_{11}^{\alpha} h_{33}^{\alpha}+h_{22}^{\alpha} h_{33}^{\alpha}\right. \\
& \left.-\frac{3}{2}\left(h_{13}^{\alpha}\right)^{2}-\frac{3}{2}\left(h_{23}^{\alpha}\right)^{2}-\frac{1}{2}\left(h_{24}^{\alpha}\right)^{2}-\frac{1}{2}\left(h_{14}^{\alpha}\right)^{2}\right] \\
& \geq \overline{R i c}_{\min }^{(\mathbf{k})}-(\mathbf{k}-2) \bar{K}_{\max }-\frac{2}{3}\left[\mathbf{k} \bar{K}_{\max }-\overline{R i c}_{\min }^{(\mathbf{k})}\right]+\sum_{\alpha}\left[\sum_{i \neq j}\left(h_{i j}^{\alpha}\right)^{2}\right. \\
& \left.+\frac{\left(\sum_{i=1}^{n} h_{i i}^{\alpha}\right)^{2}}{n-1}-S_{\alpha}-\frac{3}{2}\left(h_{13}^{\alpha}\right)^{2}-\frac{3}{2}\left(h_{23}^{\alpha}\right)^{2}-\frac{1}{2}\left(h_{24}^{\alpha}\right)^{2}-\frac{1}{2}\left(h_{14}^{\alpha}\right)^{2}\right] \\
& \geq \frac{5}{3}\left[\overline{R i c}_{\min }^{(\mathbf{k})}-\left(\mathbf{k}-\frac{6}{5}\right) \bar{K}_{\max }\right]+\frac{n^{2} H^{2}}{n-1}-S .
\end{aligned}
$$

Similarly we obtain

$$
R_{1414}+R_{2424}-\left|R_{1234}\right| \geq \frac{5}{3}\left[\overline{\operatorname{Ric}}_{\min }^{(\mathbf{k})}-\left(\mathbf{k}-\frac{6}{5}\right) \bar{K}_{\max }\right]+\frac{n^{2} H^{2}}{n-1}-S .
$$

It follows from (3.8), (3.9) and the assumption that

$$
\begin{aligned}
& R_{1313}+\lambda^{2} R_{1414}+R_{2323}+\lambda^{2} R_{2424}-2 \lambda R_{1234} \\
& \geq R_{1313}+R_{2323}-\left|R_{1234}\right|+\lambda^{2}\left(R_{1414}+R_{2424}-\left|R_{1234}\right|\right) \\
& \geq\left(1+\lambda^{2}\right)\left\{\frac{5}{3}\left[\overline{R i c}_{\min }^{(\mathbf{k})}-\left(\mathbf{k}-\frac{6}{5}\right) \bar{K}_{\max }\right]+\frac{n^{2} H^{2}}{n-1}-S\right\} \\
& >0 .
\end{aligned}
$$

This together with Theorem B implies that $M$ is diffeomorphic to a spherical space form. In particular, if $M$ is simply connected, then $M$ is diffeomorphic to $S^{n}$. This completes the proof of Theorem 3.1.

Theorem 3.2. Let $M$ be a 3-dimensional complete submanifold in a Riemannian manifold $N^{3+p}$ with codimension $p(\geq 0)$. If

$$
S<\overline{\operatorname{Ric}}_{\min }^{(\mathbf{k})}-(\mathbf{k}-2) \bar{K}_{\max }+\frac{9}{2} H^{2}
$$

for some integer $\mathbf{k} \in[2,2+p]$, then $M$ is diffeomorphic to a spherical space form or $\mathbb{R}^{3}$.

Proof. When $p=0$, the assertion follows from the theorems due to Hamilton [9] and Schoen and Yau [13].

When $p \geq 1$, for any unit tangent vector $u \in U_{x} M$ at $x \in M$, we choose an orthonormal three-frame $\left\{e_{1}, e_{2}, e_{3}\right\}$ such that $e_{3}=u$. From (2.1), (2.5) and (3.7) 
we obtain

$$
\begin{aligned}
\operatorname{Ric}(u) & =R_{1313}+R_{2323} \\
& \geq \overline{R i c}_{\min }^{(\mathbf{k})}-\sum_{A=3}^{\mathbf{k}+1} \bar{R}_{A 3 A 3}+\sum_{\alpha}\left[h_{11}^{\alpha} h_{33}^{\alpha}+h_{22}^{\alpha} h_{33}^{\alpha}-\left(h_{13}^{\alpha}\right)^{2}-\left(h_{23}^{\alpha}\right)^{2}\right] \\
& \geq \overline{\operatorname{Ric}}_{\min }^{(\mathbf{k})}-(\mathbf{k}-2) \bar{K}_{\max } \\
& +\sum_{\alpha}\left[\sum_{i \neq j}\left(h_{i j}^{\alpha}\right)^{2}+\frac{\left(\sum_{i=1}^{3} h_{i i}^{\alpha}\right)^{2}}{2}-S_{\alpha}-\left(h_{13}^{\alpha}\right)^{2}-\left(h_{23}^{\alpha}\right)^{2}\right] \\
& \geq \overline{R i c}_{\min }^{(\mathbf{k})}-(\mathbf{k}-2) \bar{K}_{\max }+\frac{9}{2} H^{2}-S .
\end{aligned}
$$

This together with the assumption implies that $R i c_{M}>0$. It follows from the theorems of Hamilton [9] and Schoen and Yau [13 that $M$ is diffeomorphic to a spherical space form or $\mathbb{R}^{3}$. This proves Theorem 3.2.

Proof of Theorem 1.1. From (3.4), (3.10), (3.11) and the assumption, it follows that there exists an $\epsilon>0$ such that $R i c_{\min } \geq \frac{n-1}{2} R i c_{\min }^{(2)} \geq \epsilon$. By the classical Myers theorem, we know that $M$ is compact. This together with Theorems 3.1 and 3.2 implies $M$ is diffeomorphic to a spherical space form. In particular, if $M$ is simply connected, then $M$ is diffeomorphic to $S^{n}$. This completes the proof of Theorem 1.1.

Proof of Theorem 1.2. When $p=0$, the assertion follows from Theorem 1.1.

When $p \geq 1$, setting $S_{\alpha}:=\sum_{i, j=1}^{n}\left(h_{i j}^{\alpha}\right)^{2}$, we have

$$
\left(\sqrt{2} h_{t t}^{\alpha}+\sum_{i \neq t} h_{i i}^{\alpha}\right)^{2} \geq(n-1)\left[\sum_{i=1}^{n}\left(h_{i i}^{\alpha}\right)^{2}+\sum_{i \neq j}\left(h_{i j}^{\alpha}\right)^{2}-S_{\alpha}\right]
$$

Note that for distinct $t, s, l$

$$
\begin{aligned}
\left(\sqrt{2} h_{t t}^{\alpha}+\sum_{i \neq t} h_{i i}^{\alpha}\right)^{2} & \leq(n-1)\left[\left(\frac{h_{t t}^{\alpha}}{\sqrt{2}}+h_{s s}^{\alpha}\right)^{2}+\left(\frac{h_{t t}^{\alpha}}{\sqrt{2}}+h_{l l}^{\alpha}\right)^{2}+\sum_{i \neq t, s, l}\left(h_{i i}^{\alpha}\right)^{2}\right] \\
& =(n-1)\left[\sum_{i=1}^{n}\left(h_{i i}^{\alpha}\right)^{2}+\sqrt{2}\left(h_{t t}^{\alpha} h_{s s}^{\alpha}+h_{t t}^{\alpha} h_{l l}^{\alpha}\right)\right] .
\end{aligned}
$$

This together with (3.12) implies

$$
h_{t t}^{\alpha} h_{s s}^{\alpha}+h_{t t}^{\alpha} h_{l l}^{\alpha} \geq \frac{1}{\sqrt{2}}\left[\sum_{i \neq j}\left(h_{i j}^{\alpha}\right)^{2}-S_{\alpha}\right]
$$

for distinct $t, s, l$. 
When $n=3$, for any unit vector $u \in U_{x} M$ at $x \in M$, we choose an orthonormal three-frame $\left\{e_{1}, e_{2}, e_{3}\right\}$ such that $e_{3}=u$. From $(2.1),(2.5)$ and (3.13) we obtain

$$
\begin{aligned}
\operatorname{Ric}(u) & =R_{1313}+R_{2323} \\
& \geq \overline{\operatorname{Ric}}_{\min }^{(\mathbf{k})}-\sum_{A=3}^{\mathbf{k}+1} \bar{R}_{A 3 A 3}+\sum_{\alpha}\left[h_{11}^{\alpha} h_{33}^{\alpha}+h_{22}^{\alpha} h_{33}^{\alpha}-\left(h_{13}^{\alpha}\right)^{2}-\left(h_{23}^{\alpha}\right)^{2}\right] \\
& \geq \overline{\operatorname{Ric}}_{\min }^{(\mathbf{k})}-(\mathbf{k}-2) \bar{K}_{\max }+\sum_{\alpha}\left[\frac{\sum_{i \neq j}\left(h_{i j}^{\alpha}\right)^{2}-S_{\alpha}}{\sqrt{2}}-\left(h_{13}^{\alpha}\right)^{2}-\left(h_{23}^{\alpha}\right)^{2}\right] \\
& \geq \frac{5}{3}\left[\overline{\operatorname{Ric}}_{\min }^{(\mathbf{k})}-\left(\mathbf{k}-\frac{6}{5}\right) \bar{K}_{\max }\right]-\frac{S}{\sqrt{2}} .
\end{aligned}
$$

When $n \geq 4$, suppose $\left\{e_{1}, e_{2}, e_{3}, e_{4}\right\}$ is an orthonormal four-frame and $\lambda \in \mathbb{R}$. From $(2.1),(2.5),(2.9),(3.5)$ and (3.13) we get

$$
\begin{aligned}
& R_{1313}+R_{2323}-\left|R_{1234}\right| \\
& \geq \overline{R i c}_{\min }^{(\mathbf{k})}-\sum_{A=3}^{\mathbf{k}+1} \bar{R}_{A 3 A 3}-\frac{2}{3}\left(\bar{K}_{\max }-\bar{K}_{\min }\right)+\sum_{\alpha}\left[h_{11}^{\alpha} h_{33}^{\alpha}+h_{22}^{\alpha} h_{33}^{\alpha}\right. \\
& \left.-\frac{5}{4}\left(h_{13}^{\alpha}\right)^{2}-\frac{5}{4}\left(h_{23}^{\alpha}\right)^{2}-\left(h_{24}^{\alpha}\right)^{2}-\left(h_{14}^{\alpha}\right)^{2}\right] \\
& \geq \overline{\operatorname{Ric}}_{\min }^{(\mathbf{k})}-(\mathbf{k}-2) \bar{K}_{\max }-\frac{2}{3}\left[\mathbf{k} \bar{K}_{\max }-\overline{R i c}_{\min }^{(\mathbf{k})}\right] \\
& +\sum_{\alpha}\left[\frac{\sum_{i \neq j}\left(h_{i j}^{\alpha}\right)^{2}-S_{\alpha}}{\sqrt{2}}-\frac{5}{4}\left(h_{13}^{\alpha}\right)^{2}-\frac{5}{4}\left(h_{23}^{\alpha}\right)^{2}-\left(h_{24}^{\alpha}\right)^{2}-\left(h_{14}^{\alpha}\right)^{2}\right] \\
& \geq \frac{5}{3}\left[\overline{\operatorname{Ric}}_{\min }^{(\mathbf{k})}-\left(\mathbf{k}-\frac{6}{5}\right) \bar{K}_{\max }\right]-\frac{S}{\sqrt{2}} .
\end{aligned}
$$

Similarly we get

$$
R_{1414}+R_{2424}-\left|R_{1234}\right| \geq \frac{5}{3}\left[\overline{\operatorname{Ric}}_{\min }^{(\mathbf{k})}-\left(\mathbf{k}-\frac{6}{5}\right) \bar{K}_{\max }\right]-\frac{S}{\sqrt{2}} .
$$

From (3.15) and (3.16) we obtain

$$
\begin{aligned}
& R_{1313}+\lambda^{2} R_{1414}+R_{2323}+\lambda^{2} R_{2424}-2 \lambda R_{1234} \\
& \geq R_{1313}+R_{2323}-\left|R_{1234}\right|+\lambda^{2}\left(R_{1414}+R_{2424}-\left|R_{1234}\right|\right) \\
& \geq\left(1+\lambda^{2}\right)\left\{\frac{5}{3}\left[\overline{R i c}_{\min }^{(\mathbf{k})}-\left(\mathbf{k}-\frac{6}{5}\right) \bar{K}_{\max }\right]-\frac{S}{\sqrt{2}}\right\} .
\end{aligned}
$$

From (3.14), (3.17) and the assumption, it follows that there exists an $\epsilon>0$ such that $R i c_{\min } \geq \frac{n-1}{2} R i c_{\min }^{(2)} \geq \epsilon$. By the classical Myers theorem, we know that $M$ is compact. Moreover, we have $\operatorname{Ric}_{M}>0$ when $n=3$ and $R_{1313}+\lambda^{2} R_{1414}+R_{2323}+$ $\lambda^{2} R_{2424}-2 \lambda R_{1234}>0$ when $n \geq 4$. This together with Hamilton's theorem [9] and Theorem B implies $M$ is diffeomorphic to a spherical space form. In particular, if $M$ is simply connected, then $M$ is diffeomorphic to $S^{n}$. This proves Theorem 1.2.

The following sphere theorem for 3-dimensional submanifolds follows from the proof of Theorem 1.2 and Schoen-Yau's theorem [13]. 
Theorem 3.3. Let $M$ be a 3-dimensional complete submanifold in a $(3+p)$ dimensional Riemannian manifold $N^{3+p}$ with codimension $p(\geq 0)$. If

$$
S<\sqrt{2}\left[\overline{\operatorname{Ric}}_{\min }^{(\mathbf{k})}-(\mathbf{k}-2) \bar{K}_{\max }\right]
$$

for some integer $\mathbf{k} \in[2,2+p]$, then $M$ is diffeomorphic to a spherical space form or $\mathbb{R}^{3}$.

Proof of Theorem 1.3. When $n=3$, the assertion follows from the assumption and Hamilton's theorem 9 .

When $n \geq 4$, we take $p=0$ and $\mathbf{k}=n-1$ in Theorem 3.1 and obtain that $M$ is diffeomorphic to a spherical space form. In particular, if $M$ is simply connected, then $M$ is diffeomorphic to $S^{n}$. This completes the proof of Theorem 1.3.

Corollary 3.4. Let $M^{n}$ be an $n$-dimensional, $n \geq 3$, compact Riemannian manifold. If the sectional curvature satisfies $K_{M} \leq 1$ and the Ricci curvature satisfies Ric $_{M}>n-\frac{11}{5}$, then $M$ is diffeomorphic to a spherical space form. In particular, if $M$ is simply connected, then $M$ is diffeomorphic to $S^{n}$.

Proof. From $K_{M} \leq 1$ and $R i c_{M}>n-\frac{11}{5}$, we obtain $R i c_{\min }>\left(n-\frac{11}{5}\right) K_{\max }$, which together with Theorem 1.3 completes the proof.

Example 3.5. W. Ziller 21] constructed a family of metrics $g_{s}$ on $M^{n}=S^{2 m+1}=$ $U(m+1) / U(m)$. This family of metrics $g_{s}$ has

$$
K_{\max }=\frac{(m+1) s^{2}}{2 m}, \quad K_{\min }=4-\frac{3(m+1)}{2 m} s^{2}
$$

for $s \geq \sqrt{\frac{2 m}{m+1}}$. By a calculation of the Ricci curvature due to Shen [14, we have

$$
R i c_{\min }=\left(2-\frac{s^{2}}{m}\right)(m+1) \text { for } s \geq \sqrt{\frac{2 m}{m+1}} .
$$

Note that $g_{s}$ is the standard metric on $S^{2 m+1}$ for $s=\sqrt{\frac{2 m}{m+1}}$. Then

$$
\begin{gathered}
\tau_{1}(s):=\frac{R i c_{\min }}{2 m K_{\max }}=\frac{2}{s^{2}}-\frac{1}{m}, \\
\tau_{2}(s):=\frac{K_{\min }}{K_{\max }}=\frac{8 m}{(m+1) s^{2}}-3 .
\end{gathered}
$$

When $\sqrt{\frac{2 m}{m+1}} \leq s<\sqrt{\frac{2 m}{1+m \tau_{n}}}$, we see that $\tau_{1}(s)>\tau_{n}$. This means that $g_{s}$ satisfies the pinching condition in Theorem 1.3. In particular, taking $n=5$, i.e., $m=2$, and $s$ close to $\sqrt{\frac{2 m}{1+m \tau_{n}}}$, we get that $\tau_{2}(s)$ is close to $\frac{4\left(1+m \tau_{n}\right)}{m+1}-3=\frac{1}{5}$. In this case, $g_{s}$ does not posses $\frac{1}{4}$-pinched curvature in the pointwise sense. Therefore, Theorem 1.3 is nontrivial. 
By a direct computation, we have the normalized Ricci curvatures of the compact rank one symmetric spaces (CROSS) with standard metrics:

$$
\begin{aligned}
& \operatorname{Ric}_{0}\left(\mathbb{C} P^{m}\right)=\frac{m+1}{4 m-2}, \quad \operatorname{dim}_{\mathbb{R}}\left(\mathbb{C} P^{m}\right)=2 m, \quad m \geq 2 ; \\
& \operatorname{Ric}_{0}\left(\mathbb{H} P^{m}\right)=\frac{m+2}{4 m-1}, \quad \operatorname{dim}_{\mathbb{R}}\left(\mathbb{H} P^{m}\right)=4 m, \quad m \geq 2 ; \\
& \operatorname{Ric}_{0}\left(\mathbb{O} P^{2}\right)=\frac{3}{5}, \quad \operatorname{dim}_{\mathbb{R}}\left(\mathbb{O} P^{2}\right)=16 .
\end{aligned}
$$

Motivated by Theorem 3 and the computation above, we would like to propose the following conjecture.

Conjecture A. Let $M^{n}(n \geq 4)$ be a compact Riemannian manifold. If Ric $c_{\min }>$ $\frac{3}{5}(n-1) K_{\max }$, then $M$ is diffeomorphic to a spherical space form. In particular, if $M$ is simply connected, then $M$ is diffeomorphic to $S^{n}$.

When $n=4$, Theorem 1.3 provides an affirmative answer to Conjecture A. We would also like to propose the following:

Conjecture B. Let $M^{n}(n \geq 4)$ be an even dimensional complete and simply connected Riemannian manifold such that $K_{M} \leq 1$, Ric ${ }_{M} \geq(n-1) \sigma_{n}$, where

$$
\sigma_{n}= \begin{cases}\frac{n+2}{4(n-1)} & \text { for } n=4 \text { or } 4 k+2, \quad k \geq 2, \\ \frac{n+8}{4(n-1)} & \text { for } n=4 k \geq 8, \quad k \neq 4, \\ \frac{3}{5} & \text { for } n=16 .\end{cases}
$$

Then $M$ is either diffeomorphic to $S^{n}$ or isometric to a compact rank one symmetric space.

\section{ACKNOWLEDGEMENTS}

The authors would like to thank Professors Kefeng Liu and Fangyang Zheng for their helpful discussions and valuable suggestions.

\section{REFERENCES}

1. M. Berger, Riemannian geometry during the second half of the twentieth century, University Lecture Series, Vol. 17, American Mathematical Society, Providence, RI, 2000. MR1729907 (2000h:53002)

2. C. Böhm and B. Wilking, Manifolds with positive curvature operators are space forms, Ann. of Math. (2), 167 (2008), 1079-1097. MR2415394 (2009h:53146)

3. S. Brendle, A general convergence result for the Ricci flow in higher dimensions, Duke Math. J., 145 (2008), 585-601. MR2462114 (2010a:53132)

4. S. Brendle, Ricci Flow and the Sphere Theorem, Graduate Studies in Mathematics, Vol. 111, Americam Mathematical Society, 2010. MR2583938

5. S. Brendle and R. Schoen, Sphere theorems in geometry, Surveys in Differential Geometry, Vol. 13, Int. Press, Somerville, MA, 2009, 49-84. MR2537082 (2010m:53047)

6. S. Brendle and R. Schoen, Manifolds with 1/4-pinched curvature are space forms, J. Amer. Math. Soc., 22 (2009), 287-307. MR2449060 (2010a:53045)

7. S. Brendle and R. Schoen, Classification of manifolds with weakly 1/4-pinched curvatures, Acta Math., 200 (2008), 1-13. MR2386107 (2009h:53087)

8. D. Gromoll, Differenzierbare Strukturen und Metriken positiver Krümmung auf Sphären, Math. Ann., 164 (1966), 353-371. MR0196754 (33:4940)

9. R. Hamilton, Three manifolds with positive Ricci curvature, J. Differential Geom., 17 (1982), 255-306. MR664497 (84a:53050)

10. P. Hartman, Oscillation criteria for self-adjoint second-order differential systems and "principal sectional curvature”, J. Differential Equations, 34 (1979), 326-338. MR.550049|(81a:34034) 
11. G. Huisken, Contracting convex hypersurfaces in Riemannian manifolds by their mean curvature, Invent. Math., 84 (1986), 463-480. MR837523 (87f:53066)

12. P. Petersen and T. Tao, Classification of almost quarter-pinched manifolds, Proc. Amer. Math. Soc., 137 (2009), 2437-2440. MR2495279 (2010m:53046)

13. R. Schoen and S. T Yau, Complete three-dimensional manifolds with positive Ricci curvature and scalar curvature, Ann. Math. Studies, 102 (1982), 209-228. MR645740 (83k:53060)

14. Z. M. Shen, A sphere theorem for manifolds of positive Ricci curvature, Indiana Univ. Math. J., 38 (1989), 229-233. MR982579 (90h:53044)

15. Z. M. Shen, On complete manifolds of nonnegative k-th Ricci curvature, Trans. Amer. Math. Soc., 338(1993), 289-310. MR1112548 (93j:53054)

16. K. Shiohama, Sphere theorems, Handbook of Differential Geometry, Vol. 1, F. J. E. Dillen and L. C. A. Verstraelen (eds.), Elsevier Science B.V., Amsterdam, 2000. MR.1736859 (2001c:53051)

17. K. Shiohama and H. W. Xu, The topological sphere theorem for complete submanifolds, Compositio Math., 107 (1997), 221-232. MR1458750 (98i:53080)

18. H. Wu, Manifolds of partially positive curvature, Indiana Univ. Math. J., 36 (1987), 525-548. MR 905609 (88k:53068)

19. H. W. Xu and J. R. Gu, An optimal differentiable sphere theorem for complete manifolds, Math. Res. Lett., 17 (2010), 1111-1124.

20. H. W. Xu and E. T. Zhao, Topological and differentiable sphere theorems for complete submanifolds, Comm. Anal. Geom., 17 (2009), 565-585. MR2550209 (2010k:53067)

21. W. Ziller, The Jacobi equation on naturally reductive compact Riemannian homogeneous spaces, Comm. Math. Helv., 52 (1977), 573-590. MR0474145(57:13795)

Center of Mathematical Sciences, Zhejiang University, Hangzhou 310027, People's Republic of China

E-mail address: xuhw@cms.zju.edu.cn

Center of Mathematical Sciences, Zhejiang University, Hangzhou 310027, People's Republic of China

E-mail address: gujr@cms.zju.edu.cn 